\title{
Heart Rate Response to a Timed Walk and Cardiovascular Outcomes in Older Adults: The Cardiovascular Health Study
}

\author{
Saket Girotra ${ }^{a}$ Dalane W. Kitzman ${ }^{b}$ Willem J. Kop ${ }^{f}$ Phyllis K. Stein ${ }^{c}$ \\ John S. Gottdiener ${ }^{d}$ Kenneth J. Mukamal ${ }^{\mathrm{C}}$ \\ a University of lowa Hospitals and Clinics, lowa City, lowa, ${ }^{b}$ Wake Forest University Health Sciences, \\ Winston-Salem, N.C., 'Washington University School of Medicine, St. Louis, Mo., dUniversity of Maryland \\ Medical Center, Baltimore, Md., and 'Beth Israel Deaconess Medical Center, Harvard Medical School, \\ Boston, Mass., USA; ${ }^{\mathrm{f}}$ Tilburg University, Tilburg, The Netherlands
}

\section{Key Words \\ Coronary heart disease $\cdot$ Exercise $\cdot$ Heart rate $\cdot$ Elderly}

\begin{abstract}
Objectives: To determine the relationship between heart rate response during low-grade physical exertion (6-min walk) with mortality and adverse cardiovascular outcomes in the elderly. Methods: Participants in the Cardiovascular Health Study who completed a 6-min walk test were included. We used delta heart rate (difference between postwalk heart rate and resting heart rate) as a measure of chronotropic response and examined its association with (1) allcause mortality and (2) incident coronary heart disease event, using multivariable Cox regression models. Results: We included 2,224 participants (mean age $77 \pm 4$ years; $60 \%$ women; $85 \%$ white). The average delta heart rate was 26 beats/min. Participants in the lowest tertile of delta heart rate ( $<20$ beats/min) had higher risk-adjusted mortality [hazard ratio $(\mathrm{HR}) 1.18,95 \%$ confidence interval $(\mathrm{Cl}) 1.00-1.40]$ and incident coronary heart disease (HR 1.37, 95\% Cl 1.051.78) compared to subjects in the highest tertile ( $\geq 30$ beats/ $\mathrm{min}$ ), with a significant linear trend across tertiles ( $\mathrm{p}$ for trend $<0.05$ for both outcomes). This relationship was not signifi-
\end{abstract}

cant after adjustment for distance walked. Conclusion: Impaired chronotropic response during a 6-min walk test was associated with an increased risk of mortality and incident coronary heart disease among the elderly. This association was attenuated after adjusting for distance walked.

Copyright $\odot 2012$ S. Karger AG, Basel

\section{Introduction}

Previous studies have shown a strong association between an impaired heart rate response to exercise and clinical outcomes in a broad range of clinical populations [1-8]. However, chronotropic assessment in these studies requires the use of a standardized, maximal exercise treadmill test. This is particularly cumbersome among the elderly, in whom musculoskeletal disorders and comorbidities frequently limit the ability to perform a maximal exercise stress test [9].

Distance walked on a 6-min walk test is used to assess functional status in patients with a wide range of cardiopulmonary disorders [10-14]. To our knowledge, the relationship of heart rate response during the test, an easily measured parameter that requires only two pulse mea-

\section{KARGER}

Fax +4161306 1234

E-Mail karger@karger.ch

www.karger.com (c) 2012 S. Karger AG, Basel

0008-6312/12/1222-0069\$38.00/0

Accessible online at:

www.karger.com/crd
Saket Girotra, MD, SM

University of Iowa Hospitals and Clinics

Division of Cardiovascular Diseases, Department of Internal Medicine

200 Hawkins Drive, Suite 4434 RCP, Iowa City, IA 52242 (USA)

Tel. +1 319384 6376, E-Mail saket-girotra@ uiowa.edu 
surements, with cardiovascular outcomes has not been previously studied.

Accordingly, we examined the risk of all-cause mortality and cardiovascular outcomes in subjects with an impaired heart rate response during a 6-min walk test enrolled in the Cardiovascular Health Study (CHS), a prospective cohort study of determinants of cardiovascular risk in older adults sponsored by the National Heart, Lung and Blood Institute. We hypothesized that a lower increase in heart rate on the 6-min walk test would reflect chronotropic incompetence and be associated with worse outcomes later in life.

\section{Methods}

\section{Study Design}

The CHS is a prospective, community-based, longitudinal study of older adults. The main purpose of the study is to evaluate risk factors for the development and progression of cardiovascular diseases in older adults. The design of the CHS has been previously described in detail $[15,16]$. Briefly, 5,201 ambulatory, noninstitutionalized men and women who were 65 years or older were recruited during 1989-1990 from a random selection of Medicare eligibility lists in 4 US communities: Forsyth County, North Carolina (Wake Forest University School of Medicine, Winston-Salem); Sacramento County, California (University of California, Davis); Washington County, Maryland (Johns Hopkins University, Hagerstown), and Allegheny County, Pennsylvania (University of Pittsburgh, Pittsburgh). To increase participation of the African-American community, an additional 687 participants were enrolled during 1992-1993 using the same sampling and recruitment methods. Each center's institutional review committee approved the study, and all participants gave informed consent.

\section{Data Collection}

Comprehensive information on health-related variables was collected at baseline and annually thereafter in a standardized fashion from study participants. Clinic examinations were performed annually from 1989-1990 (baseline) to 1998-1999, and again in 2005-2006. Telephone contact was made annually from 1989 to 1999 and 2005 to 2006 and twice per year from 2000 to 2004 and 2006 to 2007. Standardized questionnaires were administered at a baseline home interview, at annual clinic visits and during telephone contacts, providing updated information on clinical comorbidities, medications and cardiovascular events. In addition, participants underwent echocardiography in 19941995 ( 2 years prior to the 6-min walk test) with measurement of left ventricular (LV) systolic function and assessment of valve disease. Descriptions of data collection methods, including instruments and protocols, have been reported previously [15].

\section{Six-Minute Walk Test}

At year 9, 3,400 participants attended the follow-up visit and were screened for eligibility to perform the 6-min walk test. Subjects were excluded if they had any acute medical conditions, resting heart rate $<50$ or $>110$ beats/min, seated systolic blood pres- sure $>200 \mathrm{~mm} \mathrm{Hg}$ or seated diastolic blood pressure $>110 \mathrm{~mm} \mathrm{Hg}$, resting pulse oximetry $<90 \%$, severe aortic stenosis, recent or active angina, recent myocardial infarction or revascularization (within 3 months), need for an ambulatory aid such as a wheelchair, crutches, walker or cane or if they refused to provide informed consent.

The 6-min walk test was conducted according to a standardized protocol $[17,18]$. The test was administered by trained personnel and was performed in an internal hallway with the 100foot distance marked by colored tape on the floor. After demonstration by study staff, participants were instructed to walk from one end of the hallway to the other at their own pace covering as much ground as possible. Participants were allowed to stop and rest during the test, but were instructed to resume walking as soon as they were able to do so, with the period of rest included in the 6 -min period. A mechanical counter was used to count the number of completed laps, and an electronic timer with a buzzer sounded 6 min after the walk started to signal the completion of the test.

\section{Heart Rate}

Heart rate was measured by trained staff using a Nellcor ${ }^{\circledR}$ Pulse Oximeter N-3000 and semidisposable oximeter sensors (Nellcor $\left.{ }^{\circledR} \mathrm{D}-25\right)$ attached to the index finger of the nondominant hand. Presence of good perfusion was verified, and baseline heart rate was recorded in the seated position at the end of $1 \mathrm{~min}$. Postwalk heart rate was recorded immediately after completion of the test. The distance walked was recorded in feet using the length of the hallway and the number of laps covered including the final partial lap.

We calculated delta heart rate as the postwalk heart rate minus the baseline heart rate. Since there are no previous cutoffs for delta heart rate on the 6-min walk test, we created tertiles of this variable (lowest tertile $<20$ beats/min, middle tertile $20-30$ beats/ min, highest tertile $>30$ beats $/ \mathrm{min}$ ) and also examined it as a continuous variable.

\section{Outcomes}

Our primary outcomes were (1) all-cause mortality and (2) incident coronary heart disease, defined as a combination of incident myocardial infarction or death due to atherosclerotic coronary heart disease.

Follow-up visits were conducted by telephone every 6 months and in person annually. Deaths were identified by a review of obituaries, medical records, death certificates, Centers for Medicare and Medicaid Services health care utilization database for hospitalizations and from household contacts; $100 \%$ complete followup for ascertainment of mortality status was achieved. Myocardial infarction was ascertained from hospital records and was indicated by a clinical history of cardiac symptoms, elevated cardiac enzyme levels and serial electrocardiographic changes [19]. A CHS outcome assessment committee adjudicated all outcomes including the principal cause of death. All events until 30 June 2007 were included.

\section{Statistical Analysis}

We examined the distribution of patient demographics, prevalent comorbidities and exercise test parameters according to tertiles of delta heart rate. We generated descriptive summary statistics for each category using means \pm SD for continuous variables 
and proportions for categorical variables. We also examined trends in these characteristics across tertiles of delta heart rate using the Mantel-Haenszel test of trend and linear regression as appropriate. Finally, we used Cox proportional hazards models to evaluate the relationship between delta heart rate and our study outcomes.

Candidate variables for inclusion in the models were age, sex, race, educational attainment (less than high school, high school, college or professional), smoking status (current, past, never), comorbidities [history of hypertension, diabetes, coronary artery disease, congestive heart failure, chronic obstructive pulmonary disease, cancer, presence of angina (based on the Rose angina questionnaire), arthritis], body mass index (based on height and weight measured during the office visit), use of nodal blocking drugs (current $\beta$-blocker or digoxin use), cystatin $C$ (measure of glomerular filtration rate) and echocardiographic variables [qualitative assessment of LV systolic function (normal or depressed), presence of significant aortic valve stenosis (severe impairment in aortic valve excursion)] [20, 21]. All of the above variables were assessed at the time of the year 9 follow-up visit, except echocardiographic variables, which were assessed at year 7 .

Variables for model inclusion were selected based on clinical and statistical criteria. All variables with a significant unadjusted association with the outcome $(\mathrm{p}<0.10)$ were included in the models; age, sex and race were forced into the models regardless of statistical significance. The initial model was adjusted for age, sex and race only (model 1). Subsequent models were adjusted for smoking status, body mass index, hypertension, diabetes, history of angina, coronary heart disease, congestive heart failure, chronic obstructive pulmonary disease, cancer, adjusted cystatin C level, use of nodal blocking medications, presence of LV dysfunction and educational attainment (model 2). Since the distance walked during the 6-min walk test is known to be an important determinant of subsequent cardiovascular events [13, 22], we further explored its relationship with delta heart rate. We hypothesized that the distance walked would be a mediator of the relationship between delta heart rate and outcomes. Therefore, we included the distance walked as a variable in our models (model 3). Furthermore, we examined whether the relationship between delta heart rate and outcomes differed according to the distance covered on the test by including an interaction term of delta heart rate with distance. Similarly, we also looked for interactions with age, sex and race. Analysis of time-varying covariates did not demonstrate a violation of the proportional hazards assumption.

Because of the potential pharmacological effect of $\beta$-blockers and digoxin on both baseline and postwalk heart rate, we performed sensitivity analyses where we excluded all patients who were documented as taking these medications and repeated the analyses outlined above. Next, we also examined the association between heart rate response and clinical outcomes using an alternative definition of heart rate response. This was done using a linear regression model of postwalk heart rate on baseline heart rate. From this model, we derived residual scores (difference between observed postwalk heart rate and predicted postwalk heart rate). We defined heart rate responders as those with a residual score greater than $1 \mathrm{SD}$ above the mean and all other patients as heart rate nonresponders. We assessed the relationship between heart rate responder status and clinical outcomes using the methods described above.

Heart Rate Response on 6-Min Walk Test and CV Outcomes
All $p$ values were based on 2 -sided tests and were considered statistically significant at $\mathrm{p}<0.05$. All analyses were performed using SAS version 9.2 (SAS Institute Inc., Cary, N.C., USA). The authors had full access to the data and take responsibility for the integrity of the results. The study was approved by the Institutional Review Board at the University of Iowa.

\section{Results}

Of the 3,400 subjects who attended the year 9 followup visit, 892 (26.4\%) were excluded based on predefined criteria. Of the remaining 2,508 patients, 213 (8.5\%) did not perform the 6-min walk test. As expected, these subjects were older and had a higher prevalence of comorbidities and depressed LV function (online supplementary table 1; for all online supplementary material, see www.karger.com/doi/10.1159/000338736). The reasons for not performing the 6 -min walk test in these patients are listed in online supplementary table 2 . Our final sample included 2,224 subjects who completed the 6-min walk test and had no missing data on heart rate variables. The mean age was 77.4 years; $59.6 \%$ were women, and $85.3 \%$ were white. The average distance covered during the test was $342.7 \mathrm{~m}$, with a mean delta heart rate of 26 beats/min.

Table 1 shows the baseline characteristics of our study participants according to tertiles of delta heart rate. Subjects in the lowest tertile of delta heart rate were more likely to be men, of older age, with lower body mass index, higher prevalence of smoking and on $\beta$-blockers or digoxin ( $\mathrm{p}<0.01$ for all). They also had higher baseline heart rates, achieved lower postwalk heart rates and covered shorter distances during the test compared to subjects in the highest tertile of delta heart rate $(p<0.01$ for all). The prevalence of other comorbidities did not differ significantly between the groups.

During a mean follow-up of 8.5 years, there were 998 deaths (44.9\%), with 257 (11.5\%) due to coronary heart disease. Among 2,023 participants without previous myocardial infarction, there were 241 incident cases of myocardial infarction (10.8\%). A low delta heart rate was associated with an increased risk of clinical outcomes (table 2). The risk of adjusted all-cause mortality was higher in subjects in the lowest tertile [hazard ratio (HR) 1.18, 95\% confidence interval (CI) 1.00-1.41] and middle tertile (HR 1.13, 95\% CI 0.96-1.34), with a linear trend across tertiles ( $\mathrm{p}$ for trend 0.04; table 2). Likewise, the risk of incident coronary heart disease was also higher in this group (lowest tertile: HR 1.37, 95\% CI 1.05-1.78; middle tertile: HR 1.29, 95\% CI 0.99-1.68; p for trend 0.02; ta- 
Table 1. Baseline characteristics of study participants according to tertiles of delta heart rate

\begin{tabular}{|c|c|c|c|c|}
\hline & $\begin{array}{l}\text { Lowest tertile } \\
\text { (0-19 beats } / \mathrm{min})\end{array}$ & $\begin{array}{l}\text { Middle tertile } \\
\text { (20-29 beats/min) }\end{array}$ & $\begin{array}{l}\text { Highest tertile } \\
\text { ( } \geq 30 \text { beats } / \mathrm{min})\end{array}$ & $\begin{array}{l}\mathrm{p} \text { value for } \\
\text { trend }\end{array}$ \\
\hline Number of subjects & 719 & 735 & 770 & \\
\hline \multicolumn{5}{|l|}{ Demographics } \\
\hline Age, years & $77.9 \pm 4.4$ & $77.5 \pm 4.4$ & $77.0 \pm 4.2$ & 0.003 \\
\hline Female & $396(55.1)$ & $442(60.1)$ & $501(65.1)$ & $<0.001$ \\
\hline White race & $607(84.4)$ & $618(84.1)$ & $662(86.0)$ & 0.40 \\
\hline \multicolumn{5}{|l|}{ Educational attainment } \\
\hline Up to middle school & $74(10.3)$ & $71(9.7)$ & $93(12.1)$ & 0.16 \\
\hline High school & $361(50.2)$ & $367(49.9)$ & $395(51.3)$ & \\
\hline College or professional & $284(39.5)$ & $297(40.4)$ & $282(36.6)$ & \\
\hline \multicolumn{5}{|l|}{ Comorbidities } \\
\hline Hypertension & $343(47.7)$ & $350(47.6)$ & $341(44.3)$ & 0.18 \\
\hline Diabetes & $98(13.6)$ & $102(13.9)$ & $100(13.0)$ & 0.71 \\
\hline Coronary heart disease & $154(21.4)$ & $155(21.1)$ & $134(17.4)$ & 0.051 \\
\hline Congestive heart failure & $43(6.0)$ & $39(5.3)$ & $40(5.2)$ & 0.51 \\
\hline Smoking history & $392(55.2)$ & $383(52.7)$ & $374(45.8)$ & 0.003 \\
\hline COPD & $60(8.4)$ & $66(9.0)$ & $50(6.5)$ & 0.17 \\
\hline Cancer & $42(5.9)$ & $41(5.6)$ & $41(5.4)$ & 0.67 \\
\hline Body mass index & $26.2 \pm 4.1$ & $26.9 \pm 4.3$ & $27.2 \pm 4.6$ & $<0.001$ \\
\hline Angina & $27(3.8)$ & $25(3.4)$ & $24(3.2)$ & 0.50 \\
\hline$\beta$-Blockers or digoxin & $188(26.2)$ & $156(21.2)$ & $121(15.7)$ & $<0.001$ \\
\hline Adjusted cystatin C & $1.12(0.29)$ & $1.11(0.32)$ & $1.09(0.30)$ & 0.10 \\
\hline Depressed LV function & $13(2.0)$ & $22(3.3)$ & $12(1.7)$ & 0.13 \\
\hline \multicolumn{5}{|l|}{ Exercise parameters } \\
\hline Baseline HR, beats/min & $73.7 \pm 12.3$ & $71.4 \pm 10.5$ & $69.3 \pm 10.0$ & $<0.001$ \\
\hline Postwalk HR, beats/min & $86.7 \pm 12.2$ & $95.8 \pm 10.8$ & $108.7 \pm 12.3$ & $<0.001$ \\
\hline Distance walked, m & $315.5 \pm 86.5$ & $342.0 \pm 77.4$ & $368.8 \pm 90.6$ & $<0.001$ \\
\hline
\end{tabular}

All values represent numbers of participants (\%) or means $\pm \mathrm{SD}$, as appropriate. COPD $=$ Chronic obstructive pulmonary disease; $\mathrm{HR}=$ heart rate.

ble 2). When we included distance walked in the model (model 3), the association between delta heart rate and mortality was completely eliminated. There was no interaction of the effect of delta heart rate on clinical outcomes by distance walked, age, gender, race and baseline heart rate ( $\mathrm{p}$ for all interactions $>0.20$ ). Importantly, subjects who did not complete the walk test had a significantly higher risk of mortality (adjusted HR 1.35, 95\% CI 1.041.75; p value 0.02 ) compared to those who completed the test.

In sensitivity analyses, we excluded 465 patients on nodal blocking drugs ( $\beta$-blockers or digoxin) at the time of the visit. The adjusted risk for all-cause mortality (lowest tertile: HR 1.22, 95\% CI 1.01-1.48; middle tertile: HR $1.13,95 \%$ CI 0.94-1.37; p for trend 0.04) and incident coronary heart disease (lowest tertile: HR 1.46, 95\% CI 1.081.99; middle tertile: HR 1.33 , 95\% CI 0.98-1.817; p for trend 0.02) was similar to that observed in the entire cohort. Using an alternative definition of heart rate response (residual scores derived from a regression model), we found results that were qualitatively similar to our main results (online suppl. table 3). Finally, arthritis of the hip and knee were not associated with the study outcomes, and their inclusion in the models did not materially change our findings (results not shown).

\section{Discussion}

Among a population of community-dwelling older adults, we found that a lower increase in heart rate during the 6-min walk test was associated with an increased risk of all-cause mortality and coronary heart disease outcomes, and this risk was greatest among those with the lowest increase in heart rate $(<20$ beats/min). Subjects who failed to complete the 6 -min walk test were at the 
Table 2. Relative risk of all-cause mortality and composite cardiovascular events according to delta heart rate

\begin{tabular}{|c|c|c|c|c|c|}
\hline & $\begin{array}{l}\text { Continuous heart rate } \\
\text { (per } 10 \text { beats/min } \\
\text { decrease) }\end{array}$ & $\begin{array}{l}\text { Lowest tertile } \\
(0-19 \text { beats/min) }\end{array}$ & $\begin{array}{l}\text { Middle tertile } \\
\text { (20-29 beats/min) }\end{array}$ & $\begin{array}{l}\text { Highest tertile } \\
(\geq 30 \text { beats } / \mathrm{min})\end{array}$ & $\begin{array}{l}\mathrm{p} \text { value } \\
\text { for trend }\end{array}$ \\
\hline Number of subjects & 2,224 & 719 & 735 & 770 & \\
\hline \multicolumn{6}{|l|}{ All-cause mortality } \\
\hline Number of events & $998(44.9 \%)$ & $357(49.7 \%)$ & $330(44.9 \%)$ & $311(40.4 \%)$ & \\
\hline \multicolumn{6}{|l|}{ HR } \\
\hline Model 1 & $1.06(1.01-1.11)$ & $1.19(1.02-1.39)$ & $1.11(0.95-1.25)$ & 1.00 & 0.02 \\
\hline Model 2 & $1.05(1.00-1.11)$ & $1.18(1.00-1.40)$ & $1.13(0.96-1.34)$ & 1.00 & 0.05 \\
\hline Model 3 & $1.00(0.95-1.05)$ & $0.99(0.83-1.17)$ & $1.02(0.86-1.21)$ & 1.00 & 0.85 \\
\hline \multicolumn{6}{|c|}{ Incident myocardial infarction or coronary death } \\
\hline Number of events & $409(18.4 \%)$ & $149(20.7 \%)$ & $146(19.9 \%)$ & $114(14.8 \%)$ & \\
\hline \multicolumn{6}{|l|}{ HR } \\
\hline Model 1 & $1.10(1.01-1.19)$ & $1.40(1.10-1.79)$ & $1.34(1.05-1.71)$ & 1.00 & 0.01 \\
\hline Model 2 & $1.10(1.01-1.20)$ & $1.37(1.05-1.78)$ & $1.29(0.99-1.68)$ & 1.00 & 0.02 \\
\hline Model 3 & $1.07(0.98-1.17)$ & $1.27(0.96-1.66)$ & $1.24(0.95-1.61)$ & 1.00 & 0.10 \\
\hline
\end{tabular}

Figures in parentheses represent 95\% CIs, except where indicated otherwise. Model 1 was adjusted for age, sex and race only. Model 2 was additionally adjusted for smoking status, body mass index, hypertension, diabetes, history of angina, coronary heart disease, congestive heart failure, chronic obstructive pulmonary disease, cancer, adjusted cystatin C level, use of nodal blocking drugs, presence of LV dysfunction and educational attainment. Model 3 was additionally adjusted for distance walked.

highest risk. The association with mortality was completely eliminated after adjustment for distance walked.

First reported nearly 40 years ago [7], the relationship between impaired heart rate response during exercise and clinical outcomes has been well established in a broad range of clinical populations $[2,4-6]$. Lauer et al. $[5,8]$ found a low chronotropic index during exercise to be strongly associated with all-cause mortality, even after accounting for the presence of perfusion defects on stress imaging [5] or abnormalities on stress echocardiography [8]. Other studies have shown this measure to be superior to coronary angiography in predicting clinical outcomes $[4,6]$. Our prior work has shown that an impaired heart rate response during exercise is associated with adverse cardiovascular outcomes and atherosclerotic disease progression even among patients who have undergone revascularization with coronary artery bypass graft surgery [3].

However, the above studies relied on the use of a maximal exercise treadmill test to determine chronotropic response. Given the physical limitations that come with aging, including arthritis and osteoporosis, many elderly patients are unable to complete a maximal exercise treadmill test [9]. The 6-min walk test, on the other hand, is much simpler to perform and requires only a measured hallway and stopwatch. Thus, our finding of a relationship between a low delta heart rate on the 6-min walk test

Heart Rate Response on 6-Min Walk Test and CV Outcomes carries particular relevance among older patients who may be otherwise limited in performing more sophisticated assessments like a cardiopulmonary exercise test. Moreover, our findings are consistent with a previous study that found delta heart rate on a submaximal treadmill test to be as strongly associated with mortality and coronary heart disease incidence as more sophisticated measures of chronotropic incompetence like chronotropic reserve index (which usually requires a maximal exercise test) [23]. Thus, our findings would suggest that an impaired heart rate response on the 6-min walk test can be useful in assessing the risk of adverse outcomes in this population.

The association between delta heart rate and clinical outcomes was not significant after adjustment for distance walked. This finding was not entirely unexpected, since impaired heart rate response to exercise would limit functional capacity and the total distance covered and thus may be in the causal pathway [24]. While low delta heart rate was not found to be independently associated with clinical outcomes after adjusting for total distance walked, this finding does not negate the biological association between heart rate response with exercise and clinical outcomes. Furthermore, measurement of heart rate is substantially simpler in clinical practice compared to distance walked; the latter requires a measured hall- 
way free of interference or traffic and real-time accounting of the number of laps covered during a 6-min period, which may be cumbersome in a busy clinical practice. Thus, the 6-min walk provides easily obtainable data, including ability to complete the walk and heart rate at the beginning and end, which convey important prognostic information with little inconvenience, monitoring or specialized equipment.

It is important to note that this finding was significant even after adjusting for a wide range of comorbidities including LV function. In fact, LV function was preserved in the vast majority of participants (92\%) in our study, suggesting the role of other factors in causing an impaired heart rate response. Previous studies have suggested an important role for autonomic dysregulation in mediating this relationship [25, 26]; however, the underlying mechanisms are still elusive.

The strengths of our study include a diverse, community-based, older population with extensive assessment of cardiovascular and overall health at baseline and over time, long-term follow-up and formally adjudicated clinical events. Potential limitations should also be noted. Firstly, participants were not asked to discontinue $\beta$ blockers or digoxin prior to the walk test. However, we adjusted for use of these drugs in our analysis and also performed sensitivity analyses excluding patients taking these drugs; our overall study results were not significantly changed using either of these strategies. Secondly, detailed information in the CHS allowed us to adjust for the confounding effect of a number of comorbidities that may cause functional limitation. However, the potential for residual confounding may still remain. Thirdly, since our study included only older subjects and the vast majority of them had normal LV function, these findings may not be generalizable to younger or sicker patients. Finally, we acknowledge that when feasible, a maximal exercise test may yield superior data regarding heart rate response, functional capacity and overall prognosis, particularly when combined with expired gas analysis, albeit at a higher cost.

To conclude, an impaired heart rate response on the 6 -min walk test was associated with an increased risk of death and adverse coronary outcomes among older adults. It was also associated with functional capacity as measured by the ability to walk longer distances, which appeared to account for its association with clinical events. Although delta heart rate was not superior to distance walked, it is substantially easier to measure, which would make it attractive for prognostic assessment in a busy clinical practice.

\section{Acknowledgements}

The CHS is supported by contracts N01-HC-85239, N01HC-85079 through N01-HC-85086, N01-HC-35129, N01 HC15103, N01 HC-55222, N01-HC-75150 and N01-HC-45133 and grant HL080295 from the National Heart, Lung and Blood Institute, with additional contribution from the National Institute of Neurological Disorders and Stroke. Additional support was provided through AG-023629, AG-15928, AG-20098 and AG-027058 from the National Institute on Aging. A full list of principal CHS investigators and institutions can be found at http://www.chsnhlbi.org/pi.htm. No external funds were used for this study.

\section{References}

1 Lauer M, Froelicher ES, Williams M, Kligfield P: Exercise testing in asymptomatic adults: a statement for professionals from the American Heart Association Council on Clinical Cardiology, Subcommittee on Exercise, Cardiac Rehabilitation, and Prevention. Circulation 2005; 112:771-776.

- 2 Mora S, Redberg RF, Cui Y, et al: Ability of exercise testing to predict cardiovascular and all-cause death in asymptomatic women: a 20 -year follow-up of the lipid research clinics prevalence study. JAMA 2003;290: 1600-1607.

-3 Girotra S, Keelan M, Weinstein AR, Mittleman MA, Mukamal KJ: Relation of heart rate response to exercise with prognosis and atherosclerotic progression after coronary artery bypass grafting. Am J Cardiol 2009;103 $1386-1390$.
4 Brener SJ, Pashkow FJ, Harvey SA, Marwick TH, Thomas JD, Lauer MS: Chronotropic response to exercise predicts angiographic severity in patients with suspected or stable coronary artery disease. Am J Cardiol 1995; 76:1228-1232.

5 Lauer MS, Francis GS, Okin PM, Pashkow FJ, Snader CE, Marwick TH: Impaired chronotropic response to exercise stress testing as a predictor of mortality. JAMA 1999;281: 524-529.

-6 Dresing TJ, Blackstone EH, Pashkow FJ, Snader CE, Marwick TH, Lauer MS: Usefulness of impaired chronotropic response to exercise as a predictor of mortality, independent of the severity of coronary artery disease. Am J Cardiol 2000;86:602-609.
7 Hinkle LE Jr, Carver ST, Plakun A: Slow heart rates and increased risk of cardiac death in middle-aged men. Arch Intern Med 1972;129:732-748.

-8 Lauer MS, Mehta R, Pashkow FJ, Okin PM, Lee K, Marwick TH: Association of chronotropic incompetence with echocardiographic ischemia and prognosis. J Am Coll Cardiol 1998;32:1280-1286.

9 Gulati M, McBride PE: Functional capacity and cardiovascular assessment: submaximal exercise testing and hidden candidates for pharmacologic stress. Am J Cardiol 2005;96: 11J-19J.

10 Flaherty KR, Andrei AC, Murray S, et al: Idiopathic pulmonary fibrosis: prognostic value of changes in physiology and six-minutewalk test. Am J Respir Crit Care Med 2006; 174:803-809. 
11 Miyamoto S, Nagaya N, Satoh T, et al: Clinical correlates and prognostic significance of six-minute walk test in patients with primary pulmonary hypertension. Comparison with cardiopulmonary exercise testing. Am J Respir Crit Care Med 2000;161:487-492.

$\checkmark 12$ Newman AB, Simonsick EM, Naydeck BL, et al: Association of long-distance corridor walk performance with mortality, cardiovascular disease, mobility limitation, and disability. JAMA 2006;295:2018-2026.

13 Rostagno C, Olivo G, Comeglio M, et al: Prognostic value of 6-minute walk corridor test in patients with mild to moderate heart failure: comparison with other methods of functional evaluation. Eur J Heart Fail 2003; $5: 247-252$.

14 Sciurba F, Criner GJ, Lee SM, et al: Six-minute walk distance in chronic obstructive pulmonary disease: reproducibility and effect of walking course layout and length. Am J Respir Crit Care Med 2003;167:1522-1527.

15 Fried LP, Borhani NO, Enright P, et al: The Cardiovascular Health Study: design and rationale. Ann Epidemiol 1991;1:263-276.
16 Tell GS, Fried LP, Hermanson B, Manolio TA, Newman AB, Borhani NO: Recruitment of adults 65 years and older as participants in the Cardiovascular Health Study. Ann Epidemiol 1993;3:358-366.

17 Enright PL, McBurnie MA, Bittner V, et al: The 6-min walk test: a quick measure of functional status in elderly adults. Chest 2003; $123: 387-398$.

18 CHS Data Collection Forms. Cardiovascular Health Study, Public Use Data, 2002. http:// www.chs-nhlbi.org/forms/r69p1.htm (accessed June 23, 2011).

19 Ives DG, Fitzpatrick AL, Bild DE, et al: Surveillance and ascertainment of cardiovascular events. The Cardiovascular Health Study. Ann Epidemiol 1995;5:278-285.

20 Gardin JM, Siscovick D, Anton-Culver H, et al: Sex, age, and disease affect echocardiographic left ventricular mass and systolic function in the free-living elderly. The Cardiovascular Health Study. Circulation 1995; 91:1739-1748.

21 Stewart BF, Siscovick D, Lind BK, et al: Clinical factors associated with calcific aortic valve disease. Cardiovascular Health Study. J Am Coll Cardiol 1997;29:630-634.
22 Bittner V, Weiner DH, Yusuf S, et al: Prediction of mortality and morbidity with a 6-minute walk test in patients with left ventricular dysfunction. SOLVD Investigators. JAMA 1993;270:1702-1707.

23 Lauer MS, Okin PM, Larson MG, Evans JC, Levy D: Impaired heart rate response to graded exercise. Prognostic implications of chronotropic incompetence in the Framingham Heart Study. Circulation 1996;93:1520 1526.

24 Brubaker PH, Kitzman DW: Chronotropic incompetence: causes, consequences, and management. Circulation 2011;123:10101020.

-25 De Ferrari GM, Sanzo A, Bertoletti A, Specchia G, Vanoli E, Schwartz PJ: Baroreflex sensitivity predicts long-term cardiovascular mortality after myocardial infarction even in patients with preserved left ventricular function. J Am Coll Cardiol 2007;50: 2285-2290.

26 Tsuji H, Larson MG, Venditti FJ Jr, et al: Impact of reduced heart rate variability on risk for cardiac events. The Framingham Heart Study. Circulation 1996;94:2850-2855. 